\title{
A Hair Growth-Enhancing Component of Hydrolysate of Human Placenta: In vitro and In vivo Assessments
}

\begin{abstract}
Background: Human placenta hydrolysate (HPH) hasbeen used to promote wound healing, for its anti-aging effects, and, more recently, for hair growth. However, the main component(s) of HPH that induce ha ir growth rema in unknown.

Aim: To test the hypothesis that IGF-1, a component of $\mathrm{HPH}$, promotes hair growth and to evaluate the possible mechanism.

Methods: We examined the effects of IGF-1 and an amino acid mixture on the cell viability of human demal papilla cells and on GSK-3 $\beta$ and beta-catenin signaling pathways. We also examined the effects of IGF-1 on the development of hair follicles in C57BL6/J mice using hematoxylin and eosin staining.

Results: We found that IGF-1 modulated hair growth signal. Specifically, it increased both the number and size of hair follicles in groups treated with IGF-1 and with the a mino acid mixture.

Conclusions: IGF-1 and amino acids are active components of $\mathrm{HPH}$ in stimulating hair growth activity and $\mathrm{HPH}$ could be used as a drug candidate for treatment of alopecia.
\end{abstract}

\section{Introduction}

Hair loss is a distinct condition that can be accompanied by physical, psychological or social problems. There are various causes of hair loss including aging, hormone imbalance, stress and nutritional deficiency, and it can occur in both men and women [1]

Human placenta hydrolysate (HPH) has been used to alleviate tiredness and promote wound healing, and for its anti-ageing functions. Recently, a report showed the capability of $\mathrm{HPH}$ to stimulate hair growth [2]. However, the main component(s) of $\mathrm{HPH}$ that activate hair growth have not yet been identified.

Insulin-like growth factor 1 (IGF-1), also called somatomedin C, is a hormone similar in structure to insulin. It consists of 70 amino acids in a single chain with three intramolecular disulphide bridges [3]. IGF-3 plays an important role in childhood growth and continues to have anabolic effects in adults. A synthetic analogue of IGF-1, mecasermin, is used for the treatment of impaired growth in children [4].

IGF-1 has been reported to be an effective stimulator of hair follicle development in wide type mice in vivo [5]. Also, recent research has indicated that IGF-1 and an amino acid mixture increases myogenin protein expression, resulting in increased cell differentiation and cell proliferation [6].

IGF-1 has been identified in HPH and, in the present study, we evaluated whether IGF-1 is the main component of HPH that stimulates hair growth. We evaluated the in vitro and in vivo effects

\section{Journal of}

\section{Clinical \& Investigative} Dermatology

Yeon A No ${ }^{1}$, Tae-Rin Kwon ${ }^{1}$, Hye Min Park ${ }^{2}$, Hae Jung Han ${ }^{2}$, Hyi Jeong $\mathrm{Ji}^{2}$, Yong-Jun $\mathrm{Huh}^{3}$ and Beom Joon Kim ${ }^{1 *}$

${ }^{1}$ Department of Dermatology, Chung-Ang University College of Medicine, Seoul, Korea

${ }^{2}$ Green Cross Corporation, 303 Bojeong-dong, Giheung-gu, Yongin, Korea ${ }^{3}$ GCJBP Corporation, 107, Ihyeon-ro 30beon-gil, Giheung-gu, Yongin-si, Korea

\section{*Address for Correspondence}

Beom Joon Kim, MD., Department of Dermatology, Chung-Ang University College of Medicine, Seoul, Korea 224-1 Heukseok-dong, Dongjak-ku, Seoul 156-755, Korea, Fax: +82-2-823-1049; E-mail: beomjoon@unitel.co.kr

Submission: 17 July 2015

Accepted: 06 August 2015

Published: 12 August 2015

Copyright: (c) $2015 \mathrm{Kim} \mathrm{KY}$, et al. This is an open access article distributed under the Creative Commons Attribution License, which permits unrestricted use, distribution, and reproduction in any medium, provided the original work is properly cited.

Reviewed \& Approved by: Dr. Jeff Donovan, Assistant Professor, University of Toronto, USA

of IGF-1 on hair growth by observing its actions on human dermal papilla cells (DPCs) and in an animal model.

\section{Materials and Methods}

All procedures involving animals were conducted in accordance with the guidelines of the Institutional Animal Care and Use Committee (IACUC) of Chung-Ang University in Korea (IACUC no. 13-0002).

Quantifying IGF-1 and amino acids in human placental extracts

We used $14 \mathrm{HPHs}$ that came from different lots (Laennec, human placenta hydrolysate, GCJBP Corp., Yongin, Korea). Each vial's IGF-1 and amino acid content was evaluated. IGF-1 assays were performed at GCLabs (Yongin, Korea). First, IGF-1 was separated from binding proteins on the Liaison auto-analyzer (DiaSorin, Stillwater, MN, USA). The limit of detection for the LIAISON ${ }^{\circledR}$ IGF-1 assay was 3.0 $\mathrm{ng} / \mathrm{ml}$. Subsequently, IGF-1 was assayed using a one-step sandwich chemiluminescence immunoassay (CLIA).

Analysis of amino acids in the HPH samples was performed at GCLabs (Yongin, Korea). The analysis was performed using Liquid Chromatography-Tandem Mass Spectrometry (LC-MS/MS) on an API 3200 (Applied Biosystems, CA, USA).

\section{Human dermal papilla isolation and culture}

Human dermal papilla cells (hDPCs) were purchased as primary cells (Cefobio, Seoul, Korea) and grown in Dulbecco modified Eagle medium (DMEM; Invitrogen-Gibco-BRL, Grand Island, NY, USA) supplemented with $5 \%$ fetal bovine serum (FBS; Invitrogen-Gibco$\mathrm{BRL}$ ) and $1 \%$ penicillin in a humidified environment. hDPCs were used in the third or fourth passages. 
Citation: No YA, Kwon TR, Park HM, Han HJ, Ji HJ, et al. A Hair Growth-Enhancing Component of Hydrolysate of Human Placenta: In vitro and In vivo Assessments. J Clin Investigat Dermatol. 2015;3(1): 5.

\section{Cell proliferation assay}

hDPCs were plated at a density of $1.5 \times 10^{4}$ cells/well in 96-well plates, and their proliferation was measured using a cholecystokinin (CCK)-8 assay (Dojindo, Rockville, MD, USA). Cells were treated with various concentrations of IGF-1 $(1 \mathrm{ng} / \mathrm{ml}, 3.2 \mathrm{ng} / \mathrm{ml}, 10 \mathrm{ng} /$ $\mathrm{ml}$, or $32 \mathrm{ng} / \mathrm{ml})$ or $\mathrm{HPH}(1 \%, 5 \%, 10 \%$, or $20 \%)$ for $24,48,72$ or $96 \mathrm{~h}$. To assess proliferation, CCK- 8 solution $(10 \mu \mathrm{L})$ was added to the cells in $1 \mathrm{~mL}$ DMEM, and the cells were incubated for $2 \mathrm{~h}$ at 37 ${ }^{\circ} \mathrm{C}$. Absorbance was measured at $450 \mathrm{~nm}$ using a micro plate reader (SpectraMax 340; Molecular Devices, Sunnyvale, CA, USA).

\section{Western blot analysis}

Human DPCs were prepared using lysis buffer [50 mM Tris$\mathrm{HCl}(\mathrm{pH} 8.0), 150 \mathrm{mM} \mathrm{NaCl}, 1 \mathrm{mM}$ EDTA, $1 \% \mathrm{NP}-40,0.25 \%$ deoxycholic acid] containing a protease inhibitor cocktail (Roche Molecular Biochemicals, IN, USA). Protein was quantified using a Bio-Rad DC protein assay kit II (Bio-Rad, Hercules, CA, USA), separated on $10 \%$ SDS-PAGE gel, and electrotransferred to a nitrocellulose membrane (Millipore, Billerica, MA, USA). After blocking with $5 \%$ non-fat milk in Tris-buffered saline containing 0.5\% Tween 20 (Sigma-Aldrich Co., St. Louis, MO, USA), the blot was probed with antibodies against IGFRbeta-1, beta-catenin (BD Transduction, KY, USA), phospho-PTEN, phospho-AKT, phosphoGSK-3beta, GSK-3beta, bcl-2, bax, and actin (Cell Signaling Tech., Danvers, MA) and then exposed to HRP-conjugated anti-mouse or anti-rabbit secondary antibodies. Protein expression was detected using an enhanced chemiluminescence (ECL) system (Amersham Pharmacia, Piscataway, NJ, USA). Images of the blotted membranes were obtained using an LAS-1000 lumino-image analyzer (Fuji Film, Tokyo, Japan).

\section{Immunocytochemistry}

Human DPCs (hDPCs) at $1.0 \times 10^{4}$ cells $/ 500 \mu \mathrm{L}$ per chamber were seeded into a chamber slide, serum-starved for $24 \mathrm{~h}$, and then treated with IGF-1 for $48 \mathrm{~h}$. After treatment with $4 \%$ paraformaldehyde for $10 \mathrm{~min}$ and $0.1 \%$ Triton X-100 for $5 \mathrm{~min}$, the cultured hDPCs were incubated with anti-beta-catenin antibody $(1: 500,610154, \mathrm{BD}$ Transduction, $\mathrm{KY}, \mathrm{USA})$ at $4{ }^{\circ} \mathrm{C}$ overnight and then with FITClabeled goat anti-mouse IgG (1:1000, NB720-F, Novus Biologicals, CO, USA). A 4'6-diamidino-2-phenylindole (DAPI) mounting media kit (Golden Bridge International, Inc., WA, USA) was used to counterstain the nuclei. The immunostained cells were mounted with medium containing DAPI and visualized using an Olympus FLUOVIEW FV10i confocal microscope (Olympus Optical Co., Tokyo, Japan).

\section{Hair growth activity in vivo}

A total of 40 female C57BL/6J mice, 6 weeks old (Japan SLC, Shizuoka, Japan), were divided randomly into 4 groups (6 mice/ group). Group 1 comprised normal (untreated) mice, while group 2 (vehicle) received subcutaneous (SC) injections of saline. Group 3 was the IGF-1 group ( $3.2 \mathrm{ng} / \mathrm{ml} \mathrm{IGF-1} \mathrm{administered} \mathrm{by} \mathrm{SC} \mathrm{injection).}$ Group 4 received a mixture of 15 amino acids administered by SC injection. Each mouse that had injections received treatment with either $100 \mu \mathrm{l}$ of saline, IGF-1 or the amino acid mixture into the upper back. The animals were treated twice a week for 3 weeks; control animals (Group 2) received the vehicle solution alone. The back of each mouse was examined and photographed (3000D; Canon Inc., Tokyo, Japan) 0, 16, 23, 34 and 41 days following depilation. All experiments lasted for 41 days, and the mice were then euthanized.

\section{Histological examination}

Dorsal skin biopsies were collected, fixed with $4 \%$ paraformaldehyde (PFA) and embedded in paraffin wax. The segments were cut by microtome into serial longitudinal sections 5 $\mu \mathrm{m}$ thick, in the directions of the upper and lower dermis. The sections were transferred to pre-treated slides (ProbeOn PlusTM; Fisher Scientific, Pittsburgh, PA, USA), dewaxed, and stained with H\&E. Two independent blinded observers evaluated the serial sections.

\section{Statistical analysis}

Statistical analyses of the data were performed using the Student's $\mathrm{t}$-test or multivariate ANOVA. The results are expressed as the mean \pm standard deviation of at least three independent experiments, and $p$ $<0.05$ was considered statistically significant.

\section{Results}

\section{The principle active components of human placental extracts}

Among all 14 vials of $\mathrm{HPH}, \mathrm{IGF}-1$ was present at an average concentration of $3.2 \mathrm{ng} / \mathrm{mL}$ (Table 1A). Also, we quantified 15 amino acids in each lot of HPH: glycine, alanine, aspartic acid, leucine, serine, proline, glutamic acid, lysine, threonine, arginine, valine, phenylalanine, methionine, isoleucine and tyrosine (Table 1B). These data suggest that HPHs contain a consistent amount of IGF-1 and are rich in amino acids.

Table 1: (A) The content of IGF-1 and amino acids in human placental extract. A total of $14 \mathrm{HPH}$ samples were evaluated to assess the concentration of IGF-1 and amino acids in $\mathrm{HPH}$.

(A) IGF-1 was assayed with a one-step sandwich chemiluminescence immunoassay (CLIA) after prior separation of IGF-1 from binding proteins on the Liaison auto-analyzer (DiaSorin, Stillwater, MN, USA).

\begin{tabular}{|c|c|c|}
\hline No. & Lot No. & IGF-1(ng/ml) \\
\hline 1 & 30447 & 3 \\
\hline 2 & 30448 & 3 \\
\hline 3 & 30449 & 3 \\
\hline 4 & 30450 & 3 \\
\hline 5 & 30451 & 3 \\
\hline 6 & 30452 & 3 \\
\hline 7 & 30453 & 3 \\
\hline 8 & 30454 & 3 \\
\hline 9 & 30455 & 3 \\
\hline 10 & 30456 & 3.3 \\
\hline 11 & 30457 & 3.1 \\
\hline 12 & 30458 & 3.1 \\
\hline 13 & 30459 & 3.8 \\
\hline 14 & 30460 & 4.2 \\
\hline Average & & 3.2 \\
\hline SD & & 0.37 \\
\hline $\mathrm{N}$ & & 14 \\
\hline
\end{tabular}


Citation: No YA, Kwon TR, Park HM, Han HJ, Ji HJ, et al. A Hair Growth-Enhancing Component of Hydrolysate of Human Placenta: In vitro and In vivo Assessments. J Clin Investigat Dermatol. 2015;3(1): 5.

\section{IGF-1 enhanced viability of human dermal papilla cells}

As assessed via the CCK-8 assay, cell viability was not increased when hDPCs were treated with the 15 amino acid mixture or with any of the individual 15 amino acids at the same concentration as in the HPH. However, the proliferation rate of hDPCs slightly increased in response to $32 \mathrm{ng} / \mathrm{ml} \mathrm{IGF-1} \mathrm{(Figure} \mathrm{1).} \mathrm{Concentrations} \mathrm{of} \mathrm{IGF-1}$ below $32 \mathrm{ng} / \mathrm{ml}$ did not affect proliferation. Thus, we demonstrated that IGF-1 is an activator of proliferation in hDPCs.

\section{Expression level analysis of GSK3 $\beta$ and $\beta$-catenin in human dermal papilla cells treated with IGF-1}

To evaluate the signaling mechanism underlying the induction of the anagen phase by IGF-1, we performed Western blot analysis and immunofluorescence using antibodies specific to GSK3 $\beta$ and $\beta$-catenin. Observed via Western blot, pGSK3 $\beta$ and $\beta$-catenin expression was elevated after $48 \mathrm{~h}$ of incubation with IGF-1 (Figure 2a). We performed double immunofluorescence for $\beta$-catenin (green) and DAPI (blue). Many $\beta$-catenin cells co-localized with DAPI in cells treated with IGF-1 compared with the control cells (Figure $2 \mathrm{~b}$ ). Thus, IGF-1 preferentially activated $\beta$-catenin signaling via changes in gene expression.

\section{IGF-1 enhanced hair growth in the $\mathrm{C} 57 \mathrm{BL} / 6$ mouse model}

To evaluate the effects of IGF-1 on hair growth in vivo, we randomly divided mice into 4 groups (as detailed in the Methods section). Darkening of the dorsal skin was observed after injection of IGF-1 and the amino acids mixture. On day 16 after depilation, the skin color of mice in the IGF-1 group was darker than the same area in the control mice (Figure 3). Mice in the amino acid mixture group also exhibited darkening of the skin, but the darkening occurred earlier in the IGF-1 group. On day 41 after depilation, the hair on the back of half of the mice in the IGF-1 group was in mature anagen phase. Importantly, both IGF-1 and amino acids influenced the hair cycle and hair growth of C57BL6/J mice.

Table 1: (B) Analysis of amino acids was performed using Liquid ChromatographyTandem Mass Spectrometry (LC-MS/MS) on an API 3200 (Applied Biosystems, USA).

\begin{tabular}{|c|c|c|}
\hline Amino acids & Average $(\mu \mathrm{mol} / \mathbf{L})$ & S.D \\
\hline Glycine & 22197 & 1083.3 \\
\hline Alanine & 12920.5 & 520.5 \\
\hline Aspartic acid & 12843.9 & 523 \\
\hline Leucine & 11389.5 & 436.3 \\
\hline Serine & 9325.8 & 350.1 \\
\hline Proline & 8336.4 & 395 \\
\hline Glutamic acid & 7415.3 & 362.2 \\
\hline Lysine & 6982.3 & 190.6 \\
\hline Threonine & 6269.3 & 233.2 \\
\hline Arginine & 5465.7 & 191.4 \\
\hline Valine & 5415.3 & 110.9 \\
\hline Phenylalanine & 5094.4 & 157.8 \\
\hline Methionine & 3829.7 & 3830 \\
\hline Isoleucine & 2763.8 & 125.5 \\
\hline Tyrosine & 2306.2 & 93.9 \\
\hline & & \\
\hline & & \\
\hline
\end{tabular}

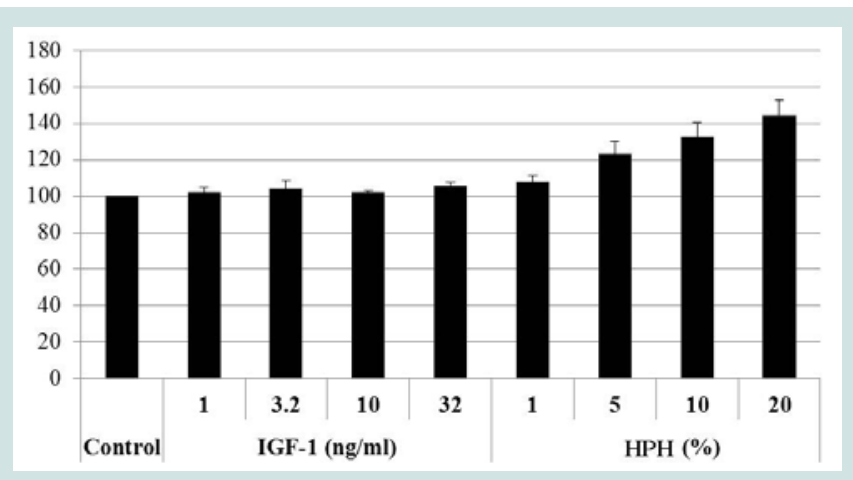

Figure 1: Effects of IGF-1 and HPH on the viability of human dermal papilla cells: Human dermal papilla cells were treated with $1 \mathrm{ng} / \mathrm{ml}, 3.2 \mathrm{ng} /$ $\mathrm{ml}, 10 \mathrm{mg} / \mathrm{ml}$ or $32 \mathrm{ng} / \mathrm{ml}$ of IGF-1 or $1 \%, 5 \%, 10 \%$ or $20 \%$ of $\mathrm{HPH}$ and incubated for $72 \mathrm{~h}$. Cell viability was measured with the CCK-8 assay. (a)

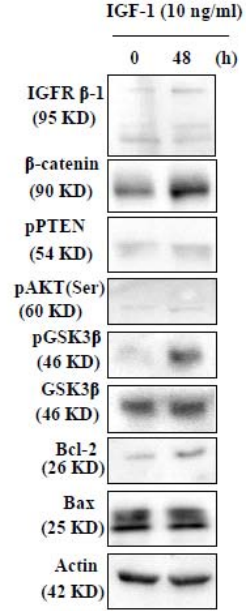

(b)

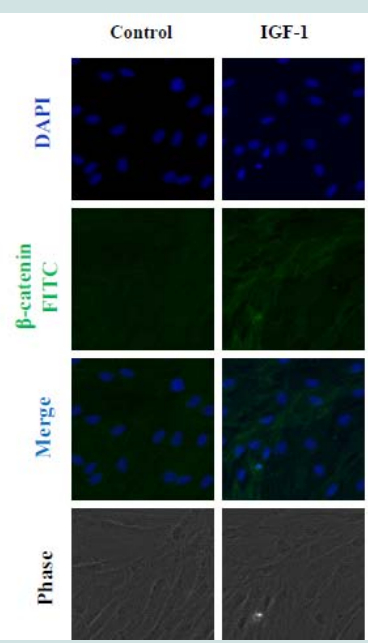

Figure 2: IGF-1 stimulates the beta-catenin signaling pathway through GSK-3beta inhibition and nuclear beta-catenin accumulation: Immunoblot was performed to assess the rate of beta-catenin expression in the harvested cells after IGF-1 treatment. (a) Western blot analysis for IGFRbeta-1, beta-catenin, phospho-PTEN, phospho-AKT, phospho-GSK3beta, GSK-3beta, bcl-2, bax, and actin in human dermal papilla cells treated with $10 \mathrm{ng} / \mathrm{ml}$ IGF-1 for $48 \mathrm{~h}$. (b) IGF-1 effectively enhanced beta-catenin activation. Representative images of immunocytochemical staining for betacatenin in hDPCs cultured with $10 \mathrm{ng} / \mathrm{ml} \mathrm{IGF-1} \mathrm{for} 48 \mathrm{~h}$. Immunocytochemical staining was carried out with the anti-beta-catenin (green) antibody. Cells were counterstained with DAPI (blue) prior to mounting. The stained cells were visualized by an Olympus FLUOVIEW FV10i confocal microscope at $\times 400$ magnification.

H\&E-stained skin tissues obtained 41 days after depilation were used to observe the status of the hair follicles and hair growth by optical microscopy. On day 41, the normal group demonstrated partial, weak development of hair roots and areas of deficiency in the inner root sheath. On the other hand, those treated with IGF-1 or the amino acid mixture had well-developed hair follicles and inner root sheaths, as well as increased hair growth (Figure 4).

\section{Discussion}

Various causes, including aging, hormone imbalance, stress and nutritional deficiency, all of which can result in hair loss in both 


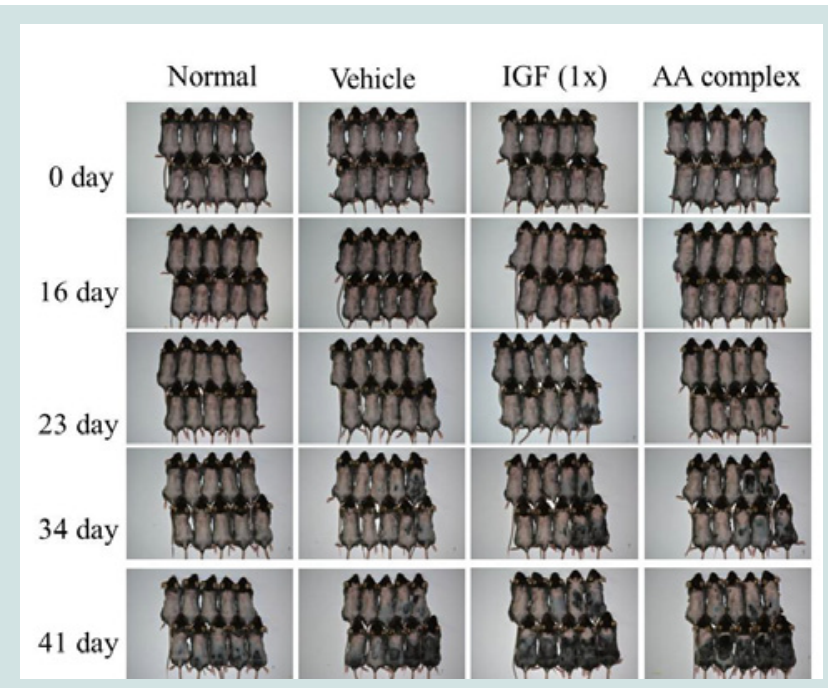

Figure 3: The growth effect of IGF-1 on the hair of mice. The hair of C57BL/6J mice was photographed at $0,16,23,34$ and 41 days after depilation. The skin on the back of each 6-week-old female C57BL/6J mouse $(n=10)$ was shaved and then the mouse was treated twice per week for 3 weeks with the experimental treatment. Treatments were injected subcutaneously into the 4-part center of the upper back and consisted of saline $(100 \mu \mathrm{l}), 3.2 \mathrm{ng} / \mathrm{ml}$ IGF-1, or an amino acid mixture (the same content as in the HPH samples). A $4^{\text {th }}$ experimental group received no injections and served as a control.

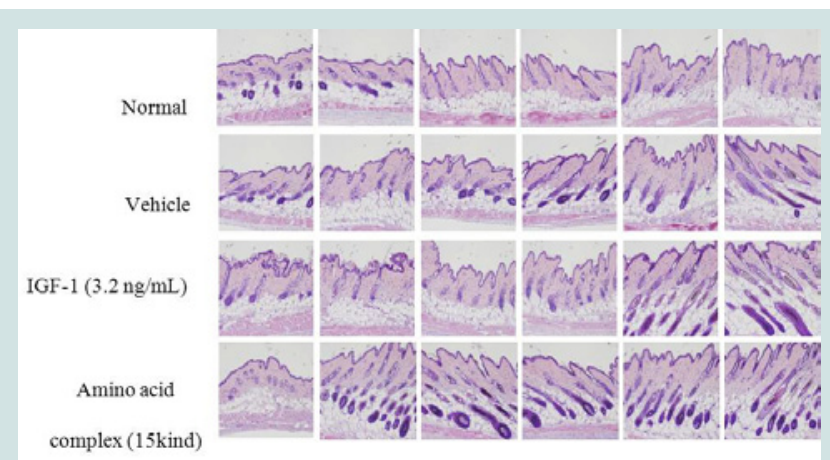

Figure 4: Hematoxylin and eosin stain of the animal model. Effects of IGF-1 and the amino acid mixture on the hair follicles of C57BL/6J mice. A representative histology result from 10 animals is shown; panels show longitudinal sections. Magnification $\times 200$ ).

men and women $[7,8]$. Several hair loss drugs have been evaluated in clinical trials and have been approved by the FDA including dihydrotestosterone-suppressing $5 \alpha$-reductase inhibitor (finasteride) and antihypertensive potassium channel opener (minoxidil). However, the efficacies of these two medications are limited and transient, owing to their unpredictable efficacies, side effects, and resumption of hair loss upon discontinuation [9].

As an alternative to oral finasteride and topical minoxidil, human placental extract (HPH), a potent hair growth modulator, has been investigated as a promising candidate for treatment of hair loss based on in vitro and in vivo studies $[2,10]$. Interestingly, a previous report showed that cow placenta extract was able to promote hair growth in C57BL/6 mice by elongating hair shafts and increasing hair follicle number [11].
Jingjie Li et al. suggested that IGF-1 increases the number of hair follicles and prolongs the growth phase. Also, in another study, IGF-1 stimulated the proliferation of follicle cells in the hair matrix into the anagen phase and down-regulated TGF- $\beta 1$ expression in hair follicles in a dose-dependent manner [5]. According to Panchaprateep R et al. DP cells from balding scalp follicles secrete significantly less IGF-1, IGFBP-2 and IGFBP-4 $(\mathrm{P}<0.05)$ than their non-balding counterparts and confirmed that the downregulation of IGF-1 may be one of the important mechanisms contributing to male pattern baldness [12].

In the present study, we quantified IGF-1 in 14 different lots of $\mathrm{HPH}$ and found that it was present in a consistent concentration. $\mathrm{HPH}$ increased the proliferation of hDPCs (Figure 1), though only at $32 \mathrm{ng} / \mathrm{mL}$ and smaller concentrations. Subsequently, to elucidate the mechanism underlying the regulation of hDPC proliferation, we focused on the GSK-3beta and beta-catenin signaling pathway with low concentrations of IGF-1. GSK-3beta is a cytoplasmic serine/ threonine protein kinase known to play a central role in a variety of biological processes [13]. Importantly, the direct inhibition of GSK3beta function results in the cellular accumulation of beta-catenin. Our results show that $10 \mathrm{ng} / \mathrm{ml}$ IGF-1 caused an induction of GSK3beta phosphorylation (Ser [8]) and an accumulation of beta-catenin compared with control (Figure 2). Thus, our findings demonstrate that IGF-1 promotes hair growth and may be a primary contributor to the hair-growth stimulation effects of $\mathrm{HPH}$.

We also evaluated the effect of HPH on hair growth for 41 days in a C57BL/6 mouse model. Our results suggest that IGF-1 and amino acids stimulate hair growth by promoting the development of hair follicles (Figure 3).

H\&E-stained skin tissue from 41 days after depilation was also used to observe the status of hair growth. Histological studies showed that the IGF-1-treated group and the amino acid mixture-treated group had markedly developed hair follicles and inner root sheaths compared to the normal group (Figure 4). These observations imply that both IGF-1 and the amino acids of HPH independently promote hair growth.

Taken together, our findings suggest that IGF-1 and amino acids in HPH are active components of HPH in stimulating hair follicle development. Furthermore, IGF-1 accomplishes its effects on hair growth via the GSK-3 $\beta$ and beta-catenin signaling pathways. Further in vitro and in vivo studies of the bioactive components in $\mathrm{HPH}$ will increase our understanding of the mechanisms by which it stimulates hair growth.

In conclusion, these observations suggested that $\mathrm{HPH}$ which contain IGF-1 and amino acids is an effective promoter of the development of hair follicles and a promising drug candidates for baldness therapy.

\section{References}

1. Mulinari-Brenner F, Bergfeld WF (2001) Hair loss: an overview. Dermatol Nurs 13: 269-272, 277-278.

2. Kwon TR, Oh CT, Park HM, Han HJ, Ji HJ, et al. (2015) Potential synergistic effects of human placental extract and minoxidil on hair growth-promoting activity in C57BL/6J mice. Clin Exp Dermatol 40: 672-681.

3. Rinderknecht E, Humbel RE (1978) The amino acid sequence of human insulin-like growth factor I and its structural homology with proinsulin. J Biol Chem 253: 2769-2776. 
Citation: No YA, Kwon TR, Park HM, Han HJ, Ji HJ, et al. A Hair Growth-Enhancing Component of Hydrolysate of Human Placenta: In vitro and In vivo Assessments. J Clin Investigat Dermatol. 2015;3(1): 5.

4. Keating GM (2008) Mecasermin. BioDrugs 22: 177-188.

5. Li J, Yang Z, Li Z, Gu L, Wang Y, et al. (2014) Exogenous IGF-1 promotes hair growth by stimulating cell proliferation and down regulating TGF- $\beta 1$ in C57BL/6 mice in vivo. Growth Horm IGF Res 24: 89-94.

6. Vélez EJ, Lutfi E, Jiménez-Amilburu V, Riera-Codina M, Capilla E, et al. (2014) IGF-1 and amino acids effects through TOR signaling on proliferation and differentiation of gilthead sea bream cultured myocytes. Gen Comp Endocrinol 205: 296-304.

7. Tucker $P$ (2009) Bald is beautiful?: the psychosocial impact of alopecia areata. J Health Psychol 14: 142-151.

8. Springer K, Brown M, Stulberg DL (2003) Common hair loss disorders. Am Fam Physician 68: 93-102.
9. Burke KE (1989) Hair loss. What causes it and what can be done about it Postgrad Med 85: 52-58, 67-73, 77.

10. Bolduc C, Shapiro J (2000) Management of androgenetic alopecia. Am J Clin Dermatol 1: 151-158.

11. Zhang D, Lijuan G, Jingjie L, Zheng L, Wang C, et al. (2011) Cow placenta extract promotes murine hair growth through enhancing the insulin - like growth factor-1. Indian J Dermatol 56: 14-18.

12. Panchaprateep $R$, Asawanonda $P$ (2014) Insulin-like growth factor-1: roles in androgenetic alopecia. Exp Dermatol 23: 216-218.

13. Sur TK, Biswas TK, Ali L, Mukherjee B (2003) Anti-inflammatory and antiplatelet aggregation activity of human placental extract. Acta Pharmacol Sin 24: 187-192. 